\title{
The Design of Smart Home Gateway Based On KNX Bus
}

\author{
Guangwei Wang \\ School of Electronic Engineering, Tianjin University of \\ Technology and Education \\ Tianjin 300222, China \\ 532119472@qq.com
}

\section{Shengli $\mathrm{Lu}$}

School of Electronic Engineering, Tianjin University of Technology and Education

Tianjin 300222, China

LSL5612@163.com

\begin{abstract}
S mart home gateway is the key to external access networks and smart home network connection device, and it can accept signals from different external networks, transmitting signals to an smart home device via home network to control and manage a home network internal device, introduces the technical characteristics and advantages on the basis of the KNX bus and combined with the latest microcontroller technology and network communication technology etc, puts forward the design of smart home gateway based on KNX bus, Described in detail the smart home gateway in Ethernet interface, KNX module and the design and implementation process of main controller. The work and research will help to break the monopoly of the KNX technology which is controlled by foreign companies and greatly reduce price of KNX product. Besides, the localization process of KNX technology and products will be effectively promoted. Ultimately, it is achieved that the smart home comes into the lives of people.
\end{abstract}

Keywords-Smart Home; KNX Bus; Embedded System; Main Controller; Gateway

\section{INTRODUCTION}

KNX bus technology is open, interactive and good, which has been widely used in the wired smart home devices in the network. However, the smart home network is not composed of $\mathrm{KNX}$ bus and Ethernet direct communication and all kinds of wireless network, not fully included in many wireless terminal equipment and Ethernet terminal equipment, to form a smart home based on the Internet of the control network. Design of a smart home gateway based on KNX bus is necessary. The smart home gateway is the key equipment of the access network and the smart home network connection, it can convert TCP/IP into KNX protocol, so as to realize the interconnection of KNX network and Ethernet and wireless network, and achieve the smart home network control.

\author{
Dangfeng Pang \\ School of Electronic Engineering, Tianjin University of \\ Technology and Education \\ Tianjin 300222, China \\ 13682107556@163.com
}

\author{
Rongrong $\mathrm{Wu}$ \\ School of Electronic Engineering, Tianjin University of \\ Technology and Education \\ Tianjin 300222, China \\ $519564315 @ q q . c o m$
}

\section{THE KNX BUS TECHNOLOGY}

$\mathrm{KNX}$ is smart home and building control areas only open to international standards, is one of Europe's three largest bus protocol EIB, BatiBus and EHS with the development of a communication mechanis m, interoperability and openness, simple and mature sound. The KNX protocol according to the ISO/OSI seven layer protocol specification which makes it the same with the other equipment and network based on ISO/OSI protocol to communicate well.

$\mathrm{KNX}$ is a distributed system based on event control. The KNX bus system adopts serial data communication controlling, monitoring and reporting. The $\mathrm{KNX}$ data is asynchronous transmission, but the message as a whole is synchronously transmitted by increasing the start and stop bits. KNX uses CSMA/CA (Carrier Sense Multiple Access/Collision Avoidance) technology to ensure bus access without reducing the trans mission rate and colliding at the same time.

\section{SMART HOME GATEWAY OVERALL DESIGN}

\section{A. The Composition of Smart Home Gateway}

The introduction of fieldbus technology prompted the rapid development of the smart home in the direction of network control system. Intelligent information processing and intelligent control have become a key technology to build smart home network system. Embedded system with high integration and reliability, flexible use, has been widely applied in the smart home control equipment and communication network. Smart home gateway based on KNX bus is made by ARM9 S3C2440 microprocessor (master controller), TP - UART - IC KNX module and Ethernet interface, the wireless communication module, etc. System is shown in Fig. 1. KNX smart home network system is made by $\mathrm{KNX}$ bus, KNX actuators, KNX 
sensors and the corresponding smart home equipment.KNX smart home network is connected with main controller by KNX module. Main controller receives and processes all kinds of information from KNX intelligent home network device, transmission through the Ethernet interface to $\mathrm{PC}$ and the Internet, the request of the intelligent terminal equipment through wireless communication module to the main controller, NANDFLASH can save the firmware program and provide storage space when the power off. In the KNX smart home network system, the sensor will upload the information of the family environment to the main controller, and the actuator receives the bus information and control information according to the main controller, and making the smart home equipment in the corres ponding working condition.

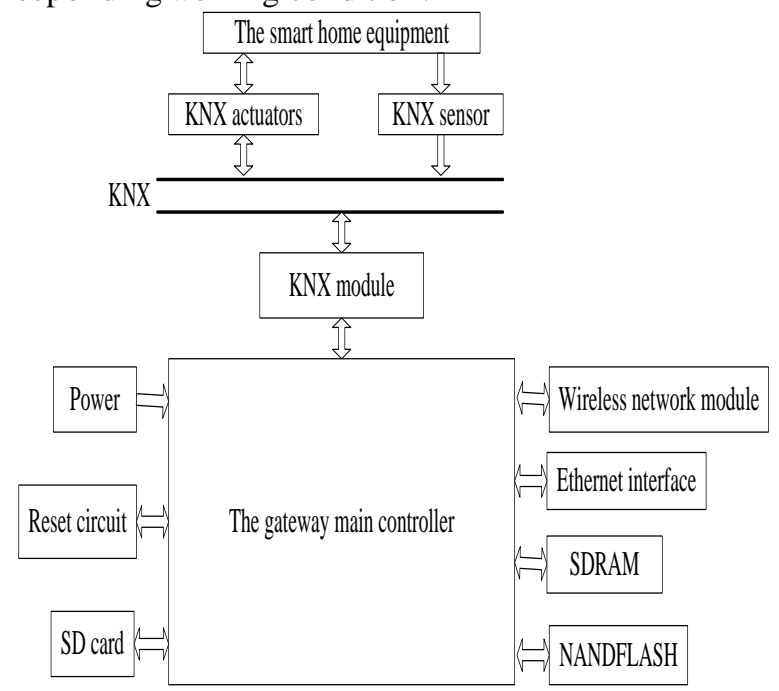

Figure 1. the composition of smart Home gateway based on KNX bus

\section{B. Smart Home Gateway Function}

The smart home gateway based on KNX bus is an important equipment to achieve the conversion between the TCP/IP protocol and $\mathrm{KNX}$ protocol, it can realize the interconnection of KNX network and Ethernet and wireless network, too. The follow are main functions:

1) Realizing the conversion between TCP/IP protocol and KNX protocol.

2) Realizing the data exchange between wireless intelligent terminal equipment (for example: WiFi, ZigBee etc.) and KNX smart Home network.

\section{THE HARDW ARE DESIGN OF SMART HOME GATEWAY}

The smart Home gateway is Transfer station between the family internal network and external network, and the gateway hardware platform is composed of main controller S3C2440ARM9, Ethernet interface, SDRAM, NANDFLASH, power / reset module and KNX module.

\section{A. The Main Controller}

The main controller is the core of s mart Ho me gateway, which main functions are communicating with the KNX module, managing the KNX network terminal equipment, completing the information interaction, between home network and KNX smart Home network etc. The system chooses the Samsung Corp S3C2440 microprocessor (ARM9 Series), the processor with high speed which uses the ARM920T kernel and the 5 stage pipeline.TheS3C2440 have varieties of peripheral interface which can extend the NANDFLASH, touch screen and a plurality of UART, it provides good support for the smart home gateway hardware design. The design of the smart home gateway hardware mainly uses the extension function of the S3C2440 microprocessor, the design of KNX interface circuit, Ethernet interface circuit, communication interface circuit, SD card interface circuit etc.

\section{B. Ethernet Interface}

The function of The Ethernet interface is to connect the main controller to the Internet, so as to realize the users' remote control and management. DM9000 and S3C2440 connection are shown in Figure 2. The module not only is a large amount of data transmission but also can support the burst data transfer quickly and large amount of access burst. Because $\mathrm{S} 3 \mathrm{C} 2440$ has been integrated in a $10 / 100 \mathrm{Mb}$ adaptive Ethernet MAC controller, simplifies the design of Ethernet circuit greatly. So the system selected the 10/100Mbps adaptive Ethernet control chip DM9000. The highest frequency of DM9000 can reach $200 \mathrm{MHZ}$, bus width up to 32 , and fully able to meet the data transmission of Ethernet 100Mbps .

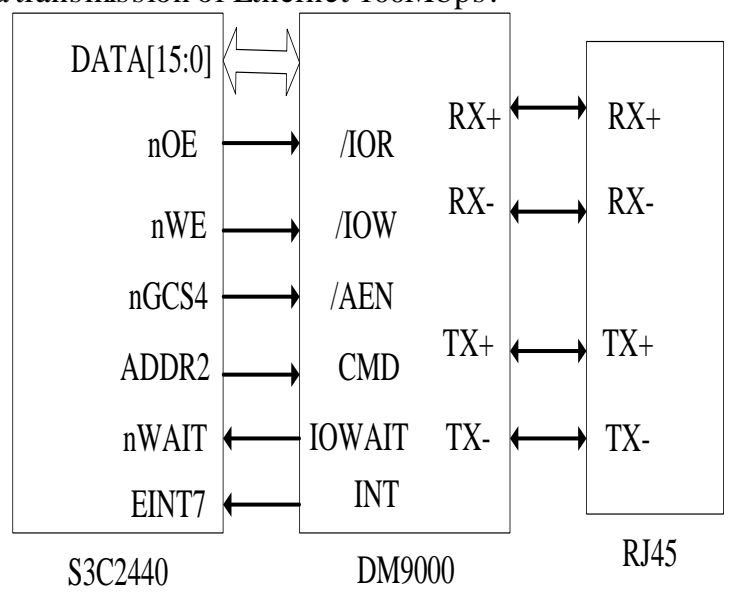

Figure 2. the connection between 2 DM9000 and S3C2440

\section{KNX Module}

The function of The KNX module is to connect the KNX s mart home network to the main controller. This system uses universal asynchronous receiver transmitter TP-UART-IC which is designed for Twisted-pair cable by Siemens. The sensor, actuator, and the micro processing controller connected to the KNX bus for transmission and reception supported by the TP-UART-IC. The TP-UARTIC and KNX bus, the S3C2440 connection is shown in Fig. 3. The main characteristics: the TP-UART-IC is composed of UATR digital interface and analog interface, the transmission rate of $9600 \mathrm{bps}$ and $19200 \mathrm{bps}$ Baud rate set by the pin TSTIN, the SA VE pin notify the microprocess or for processing when power down, MODE0 and MODE1 is normal mode by setting to "11" which are mode selection, this moment, the digital part and the analog part work. Set to " $10 "$ is the simulation model. 


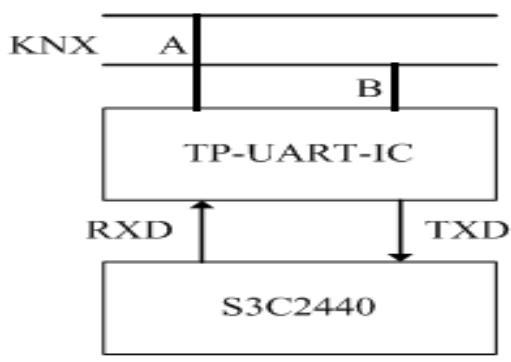

Figure 3. the connection of the TP-UART-IC, KNX bus and the $\mathrm{S} 3 \mathrm{C} 2440$

\section{THE SOFT WARE DESIGN OF SMART HOME GATEWAY}

\section{A. The Level Architecture of Software System}

The S3C2440 gateway main controller supports Linux, WINCE and other embedded operating system. The design of smart home gateway selects embedded Linux and constructs software platform, the construction of the system gets the suitable embedded Linu x kernel by cutting Linux the source code, so as to complete the software design of main controller(software architecture is shown in Fig. 4). The application layer can achieve the function of network communication, data storage and serial communication application, realizing the transformation of the TCP/IP protocol and the KNX protocol, Ethernet and wireless control realizes the control of the smart home devices, and storing all kinds of information of the smart home network, so as to query and control. The API interface function of application program calls the Linux operating system layer provide system calls and completes API interface functions, hardware driver layer completes the Ethernet driver, wireless network driver, serial port driver, SD card driver to establish a suitable environment for the Linu x operating system, According to the design of the application program operation corresponding hardware implement KNX smart home network and Ethernet etc data interaction.

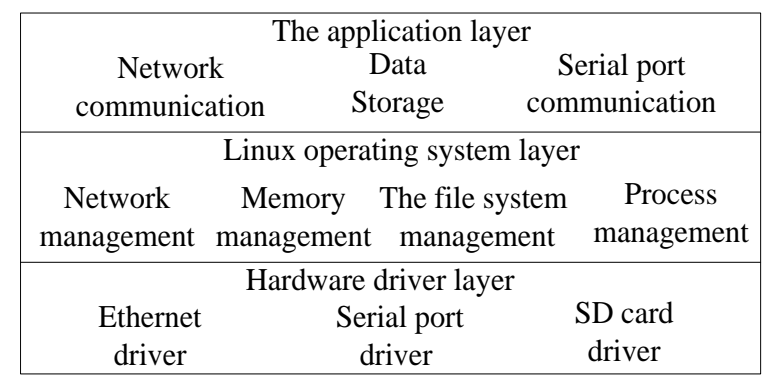

Figure 4. the level architecture of main control

\section{B. Linux Platform}

Linux is a multitasking, multi-user, free, open source operating system, it has a complete TCP/IP protocol stack, as long as a little modify the kernel code which can realize the required driver support, therefore, according to the smart home gateway design needs to select the Linux operating system. To do this, you need to carry on the
Bootloader transplant, DM9000 network card driver, serial port driver to transplant, the file systemtransplantation etc.

\section{The Main Program Design}

Main controller software process is shown in Fig. 5. After the main controller starts, the first complete hardware and software initialization, at the same time opening the serial port and the network services, and then the program is divided into two branches, and the first branch handle serial data, it also the data which comes from the KNX household network, the network data storage from the KNX home to SD card and the local database in NANDFLALSH, The second branch processing network data, network data including control, setting and query request, according to the request of the corres ponding processing.

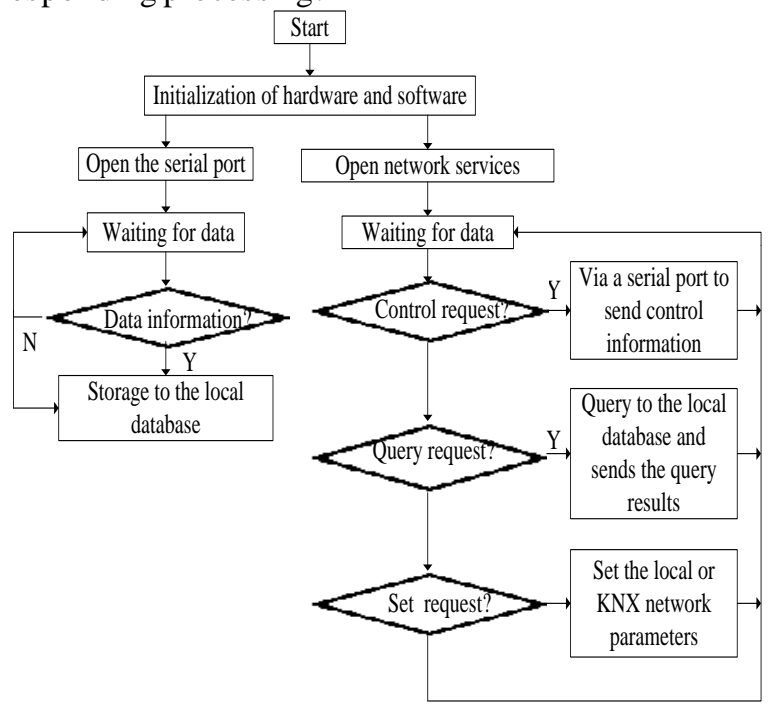

Figure 5. the main controller software flow chart

\section{TP - UART - IC Communication Program}

The TP - UART - IC processes of handler is shown in Fig. 6: after the system starts a serial port handling procedure will start automatically, serial processor will read system configuration file, opening the port configuration and getting information (including baud rate, parity bit and stop bit, etc.), and check the port whether it can open normally, such as normal open the serial port, serial port into the working state, the serial data communication in the work state is divided into two parts of the ascending and descending, the ascending module's main function is to receive data from the KNX household network information, due to the operation mode of the serial port is the current ways of working, researchers need to subcontract the received data, after receive the data from the serial port. A serial port processing module will be the first data of the subcontract, the data of the subcontract, and accuracy of the data analys is, after verification right to break up the weave a new packet of data, and then in the S3C2440's hands. Downstream processing, first of all, sent subcontract processing of data, after receive the packet. Firstly, researchers prove the validity of the data packets, verify the correctness, carry the identity of the information data, organize into a new packet, and send new packets 
through a serial port to the TP - UART - IC according to the data packet.

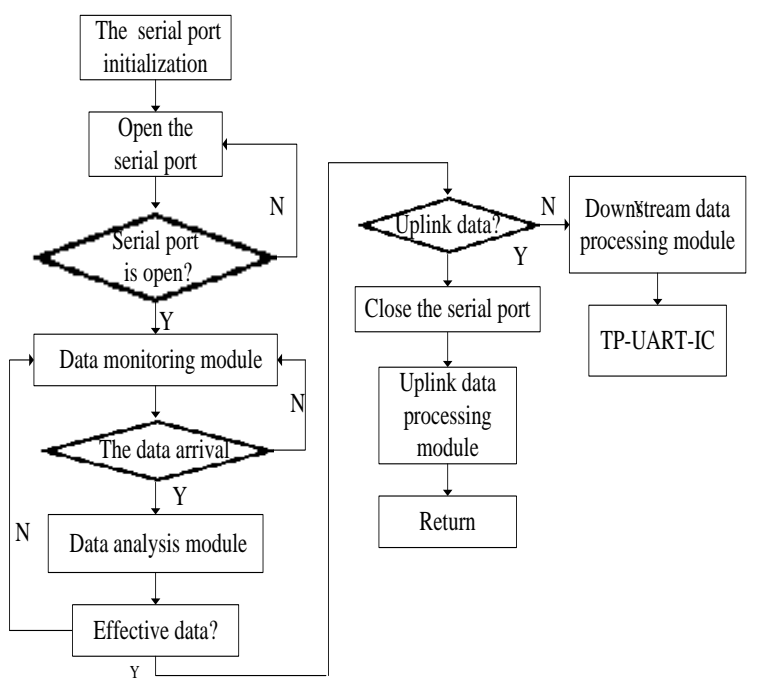

Figure 6. The process of TP - UART - IC communication

\section{E. Ethernet Communication Program}

Main controller and the Ethernet connection use Linux which supports for TCP/IP protocol, using streaming Socket communication mode. The process is shown in Fig. 7. Through the socket () system call to create a socket, and then through the bind () to bind the socket to the server port, and then call the connect () send a connection request to the server, when the connection is successful, the server side and the end of the service call the send () and recv () to send and receive data, data analys is, part of the module is similar to TP - UART - IC communication program, data processing is divided into two parts, one part is to receive Ethernet data, sending data from the Ethernet S3C2440, the other part is to send Ethernet data, send S3C2440 data to Ethernet.

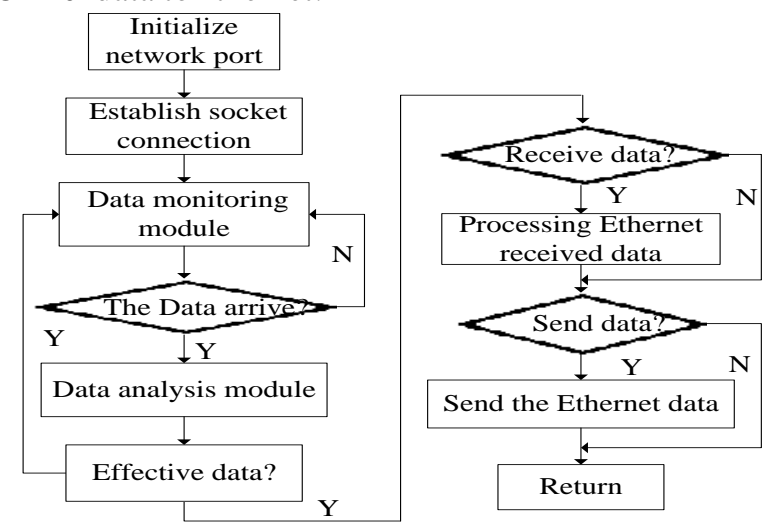

Figure 7. smart home gateway and Ethernet communication module

\section{F. Wireless Communication Program}

The communication mode between the Main controller and the wireless network adopt the asynchronous serial .The communication process as shown in Fig. 8.
First open the serial port, and determine whether a serial port normal open or not, such as open normally, read and write a serial port data, data interaction and close the serial port finally.

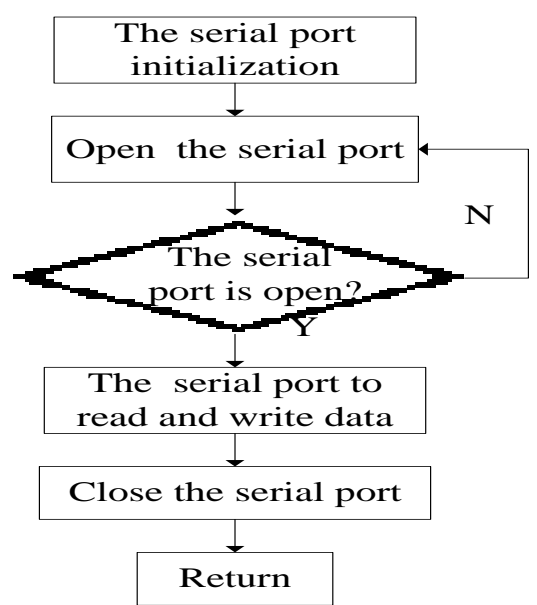

Figure 8. Smart home gateway and wireless network communication work

\section{THE CONCLUSION}

The Smart home gateway is designed completely based on KNX bus S3C2440 microprocessor as the core, it adopts the KNX module TP - UART - IC, DM9000 Ethernet adapter, and achieves the data communication between KNX smart home network and a variety of Ethernet and wireless network, so as to realize the remote control of smart home devices, information query etc. The system has low cost, low power consumption, strong practicability, good scalability, etc, and it has a wide application prospect.

\section{REFERENCES}

[1] Shuai Xue, multi-protocol gateway design and implementation of smart home system [D], Beijing university of posts and telecommunications, 2012 (1)

[2] Wen-Jun Yu, Zhi-hao Ling. Aiot of smart home system research [J], Automation instrument, 2011,32 (8): 56-59

[3] Ping Sun. The research and implementation of smart gateway based on KNX agreement [D], Nan jing university of aeronautics and astronautics, 2012 (1)

[4] Xiao-liang Huang, Xiao-hui Xu, Jun-hua Song, The design of the wireless sensor network in smart home systems [J], Journal of electronic design engineering, 2013 (04): 35 - 37

[5] Smart home. http://baike.baidu.com/view/37089.htm

[6] KNX China user organization committee. http://www.knxchina.org/home/zghy/

[7] Guo-dong Zhang, the research and application of Internet communication protocol which Based on KNX/EIB communication protocol of theory [D], Jiang nan university, 2012

[8] Shi-hui Cheng, Gui-ling Liu, Xue-ya Yang,Yue Yang,Hai-tao Xu, the design of Smart home control system based on ARM - Linux [J], Science and technology innovation herald, 2014, (5): 50-53

[9] Jian-chang Zhou, Zhen-xing Li, Based on the analysis of S3C2440 $\mathrm{u}$ - boot and transplantation [J]. Journal of electronic design engineering, 2012(17):53-56

[10] Cheng Ma, The research and design of the embedded smart home server Based on Linux [D]. 2013 (6):35-38 